Dr. med.

Ulrich

Mutschler,

Hamburg

\section{Kombinationstherapie für pädiatrische Patienten mit Vitiligo ist effektiv}

\author{
Üblicherweise wird zur Vitiligo-Therapie bei großflächigeren Depigmentie- \\ rungen UV-Licht verwendet, Tacrolimus dagegen bei geringerem Befall. Die \\ Wirksamkeit einer Kombinationstherapie wurde nun an Kindern untersucht.
}

U $\mathrm{m}$ die Kombinationstherapie zu testen, wurde eine entsprechende Pilotstudie an 20 Kindern mit Vitiligo im Alter von 4-14 Jahren von Hautärzten in Nord-Indien durchgeführt. Die Kinder hatten eine im Mittel seit 3 Jahren stabile symmetrische Vitiligo mit Befall von durchschnittlich $12 \%$ der Hautoberfläche. Die linke Seite des Körpers wurde laut Testprotokoll zweimal täglich mit $\mathrm{Ta}$ crolimus (0,03\%ige Salbe), die rechte Seite hingegen nicht behandelt. Zusätzlich erfolgte auf beiden Seiten eine langsam ansteigende Bestrahlung mit „norrow band"(NB)-UV-Licht dreimal die Woche.

Während sich bei der Monotherapie mit UV-Licht nach 2, 4 und 6 Monaten jeweils 7, 23 und $32 \%$ der Haut repigmentierten, erreichten die Stellen mit der zusätzlichen topischen Therapie 26, 46 und $72 \%$ mit einer abschließenden statistischen Signifikanz ( $p<0,001)$. Die beste Wirksamkeit konnte mit einer 100\%igen Ansprechrate (UV-Licht allein nur $65 \%$ ) mit der Kombinationstherapie im Gesicht erzielt werden, am Stamm lagen die Werte bei 60 beziehungsweise $20 \%$. Als Nebenwirkungen entwickelten zwei Patienten stärkere Rötungen, einer davon mit zusätzlicher Blasenbildung.

Dayal S et al. Treatment of childhood vitiligo using Tacrolimus ointment with narrowband ultraviolet B phototherapy. Pediatr Dermatol 2016 Oct 4

\section{Kommentar}

0,5-2\% der Weltbevölkerung haben eine Vitiligo, in Deutschland sind es circa $1 \%$ (also rund eine dreiviertel Millionen Menschen). Häufiger kommt die Erkrankung zum Beispiel in Indien und Pakistan mit bis zu $10 \%$ der Bevölkerung vor. Oft fühlen sich die Patienten ausgegrenzt oder stigmatisiert, gleichzeitig bestehen auch gelegentlich weitere Autoimmunerkrankungen wie Alopecia areata, Diabetes mellitus oder Hashimoto-Thyreoiditis. Der bekannteste Patient war Michael Jackson, der durch eine relativ aggressive Bleichung versuchte, seine Erkrankung zu verbergen.

Eine Phototherapie führt bei der Vitiligo idealerweise zur Repigmentation, zur Besserung und auch zur Vorbeugung einer weiteren Progression, und zwar infolge seiner bekannten immunsuppressiven und immunmodulatorischen Eigenschaften. UVB gibt es als Breitband- (290-320 nm), Schmalband- ("narrow band" = NB; 310-312 nm) und monochromatisches UV-Licht (MEL; 308 $\mathrm{nm}$ ). Alle drei Bereiche sind bei der Vitiligo klinisch effektiv. Der genaue Wirkmechanismus ist nicht bekannt, jedoch weiß man, dass eine spezielle UV-Bestrahlung auf spezifische Haut-Chromophoren einwirkt, mit Induktion der Apoptose, einer T-Zellverringerung, einer verringerten Antigenpräsentation sowie Downregulation von Mediatoren und Zytokinen der Inflammation. Speziell bei NB-UVB konnte gezeigt werden, dass

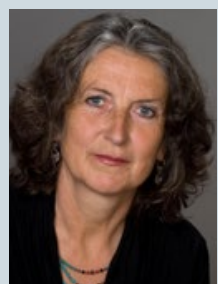

Dr. med.

Kirsten

Stollhoff,

Hamburg

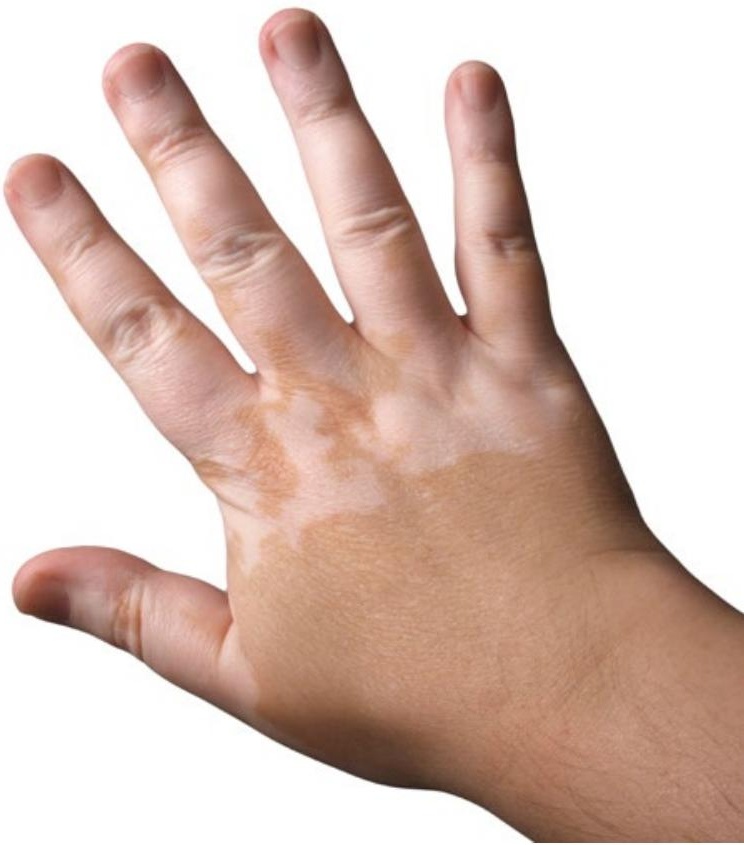

In Deutschland leidet circa $1 \%$ der Bevölkerung an Vitiligo.

hierunter die Melanozyten angeregt werden, für eine gesteigerte Melaninproduktion als auch für eine gesteigerte Migration hin zu depigmentierten Hautarealen.

Die Vorteile einer speziellen NB-UVB-Phototherapie liegen in der Vermeidung von kurzwelligeren und damit stärker erythrogenen Wellenlängen (wie z. B. des natürlichen UV-Lichtes der Sonne). Damit hat sich NBUVB als beste Wahl für eine generalisierte Vitiligo herauskristallisiert, nunmehr auch in Kombination mit Tacrolimus. Generell ist hierbei die Prognose bei weiblichen Patienten mit symmetrischer Vitiligo und ohne Beteiligung der Akren am besten. Als zusätzliche Therapiemöglichkeit existieren Transplantationen autologer epidermaler Zellsuspensionen bei kleineren Herden, PermanentTattoos bei wenig Herden mit kleiner Fläche, Selbstbräuner und vor allem auch eine unterstützende Psychotherapie.

Dr. Ulrich Mutschler 